\title{
LIN-12/Notch signaling in C. elegans ${ }^{*}$
}

\author{
Iva Greenwald ${ }^{\S}$, Howard Hughes Medical Institute and Department of \\ Biochemistry and Molecular Biophysics, Columbia University, College of \\ Physicians and Surgeons, New York, New York 10032 USA
}

\section{Table of Contents}

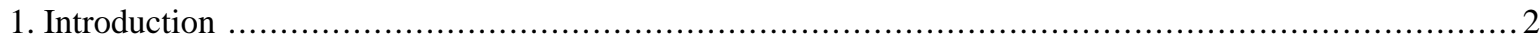

2. Genetic identification and characterization of LIN-12 and GLP-1 ..................................... 2

3. Overview of signal transduction by LIN-12 and GLP-1 .............................................. 3

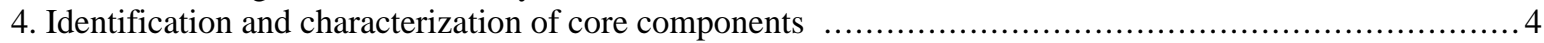

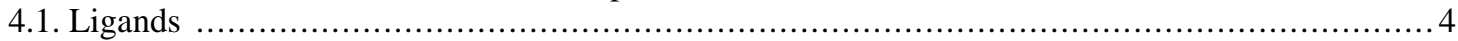

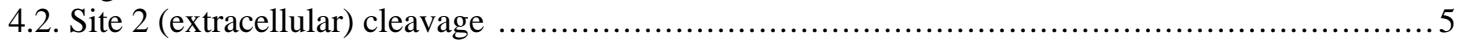

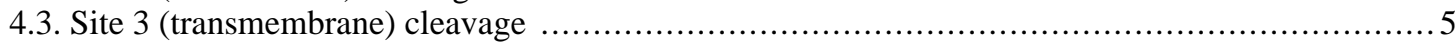

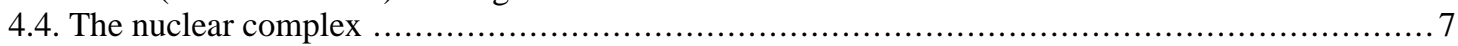

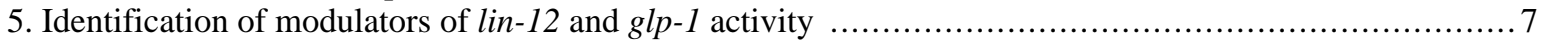

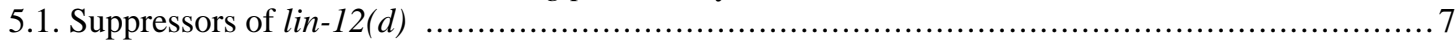

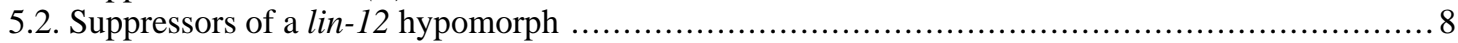

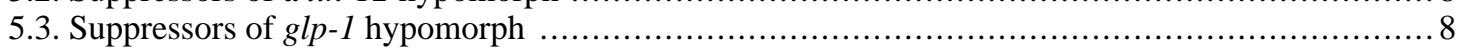

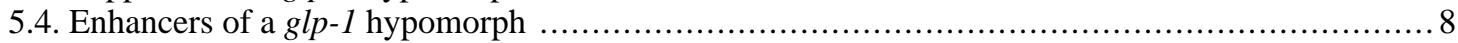

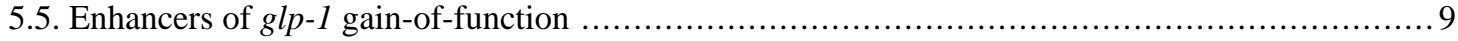

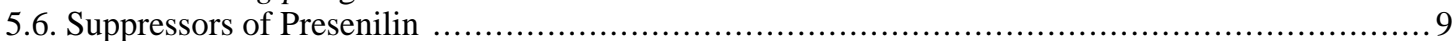

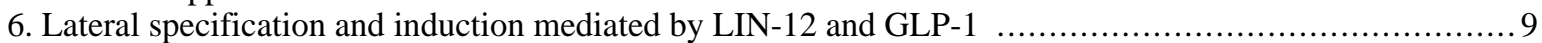

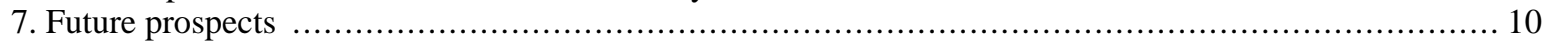

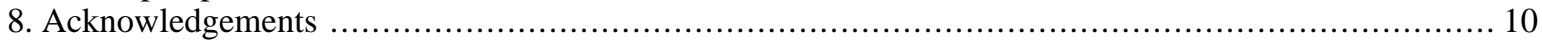

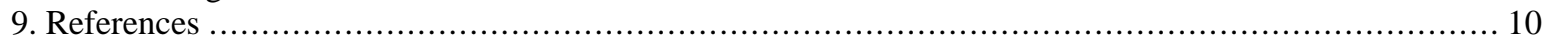

\begin{abstract}
Receptors of the LIN-12/Notch family mediate cell-cell interactions during animal development, and aberrations in LIN-12/Notch signaling have been implicated in human disease. Studies in C. elegans have been instrumental in defining the basic features of the LIN-12/Notch pathway, the role of LIN-12/Notch proteins as receptors for intercellular signals, the mechanism of signal transduction, and the regulation of LIN-12/Notch signaling during cell fate decisions. This chapter is focused on detailing how the "awesome power of $C$. elegans genetics" has identified many core components and modulators of LIN-12/Notch activity.
\end{abstract}

\footnotetext{
${ }^{*}$ Edited by Lisa R. Girard. Last revised July 29, 2005. Published August 8, 2005. This chapter should be cited as: Greenwald, I. LIN-12/Notch signaling in C. elegans (August 4, 2005), WormBook, ed. The C. elegans Research Community, WormBook, doi/10.1895/wormbook.1.10.1, http://www.wormbook.org.

Copyright: () 2005 Iva Greenwald. This is an open-access article distributed under the terms of the Creative Commons Attribution License, which permits unrestricted use, distribution, and reproduction in any medium, provided the original author and source are credited.

${ }^{\S}$ To whom correspondence should be addressed. E-mail: greenwald@ cancercenter.columbia.edu
} 


\section{Introduction}

Coming soon after the finding of a conserved 'homeobox' in developmental control genes (Laughon and Scott, 1984; McGinnis et al., 1984), the discovery that LIN-12/Notch proteins in C. elegans and Drosophila contain epidermal growth factor (EGF)-like repeats (Greenwald, 1985; Wharton et al., 1985; Kidd et al., 1986) suggested that the kinds of molecules that underlie common developmental mechanisms shared by all animals would be very broad, encompassing signals and receptors as well as DNA binding proteins, and also that basic signaling systems would be conserved. In subsequent years, systematic analysis in worms and flies has illuminated how LIN-12/Notch proteins transduce signals, and contexts in which this signaling system is used and modulated. New roles for LIN-12/Notch signaling in mammalian development and disease have been identified at a prodigious rate (Gridley, 2003; Harper et al., 2003; Hansson et al., 2004). The focus of this review will be on the contributions of genetic analysis of LIN-12/Notch signaling in C. elegans has made to illuminating universal aspects of this essential conserved pathway.

\section{Genetic identification and characterization of LIN-12 and GLP-1}

C. elegans has two LIN-12/Notch proteins, encoded by the lin-12 and glp-1 genes. These genes were identified through genetic screens for developmental mutants, and genetic analysis has established both unique and redundant roles for the two genes in specifying cell fates.

lin-12 was identified in a genetic screen for mutations that have defective vulval development (Greenwald et al.,1983; Ferguson and Horvitz,1985). The first alleles discovered, called lin-12(d) alleles, were dominant and hypermorphic, and intragenic reversion yielded null alleles, called lin-12(0) (Greenwald et al., 1983). Phenotypic analysis indicated that $\operatorname{lin}-12(d)$ and $\operatorname{lin}-12(0)$ alleles cause opposite transformations in many different cell fate decisions, including early gonadogenesis, vulval precursor cell fate specification (see Vulval development), and sex mesoblast specification (see Male development; Greenwald et al., 1983). lin-12 also is required for $\pi$ cell specification during hermaphrodite gonadogenesis (see Hermaphrodite cell-fate specification; Newman et al., 1995). Possible roles for lin-12 activity in differentiation or function, as opposed to cell fate specification per se, have also been described (Eimer et al., 2002; Chao et al., 2005). The requirement for lin-12 activity for appropriate cell movements that result in a left-right intestinal twist may also be considered as a role in cell function, rather than cell fate, between otherwise identical cells (Hermann et al., 2000).

glp- 1 was identified in two different, contemporaneous genetic screens. A screen for sterile mutants revealed that loss of 'zygotic' glp-1 activity limits germline proliferation and causes germ cells to enter meiosis prematurely (Austin and Kimble, 1987). Subsequently, dominant alleles of $g l p-1$ that cause the opposite phenotype--continued mitotic proliferation of the germline at the expense of gamete production--were identified (Berry et al., 1997; Pepper et al., 2003). A screen for maternal-effect embryonic lethal mutations revealed that loss of maternal glp- 1 activity prevents induction of the anterior pharynx at the 12-cell stage of embryogenesis (Priess et al., 1987). Subsequent experiments revealed another role for $g l p-1$ maternal activity in mediating a critical inductive event at the 4-cell stage of embryogenesis (Mango et al., 1994; Mello et al., 1994; Hutter and Schnabel, 1994; Moskowitz et al., 1994), and at least four other interactions in the early embryo (see also Notch signaling in the C. elegans embryo).

Double mutants lacking both $l i n-12$ and $g l p-1$ activity revealed that the two genes are functionally redundant in some cell fate decisions (Lambie and Kimble, 1991). The distinctive phenotype of the lin-12 glp-1 double is called Lag, for "Lin-12 and Glp-1". The hallmarks of the Lag phenotype are the absence of a rectum, anus and excretory cell, as well as a duplication of the excretory pore, reflecting defects in cell fate decisions that occur during embryogenesis (Lambie and Kimble, 1991; see also Notch signaling in the C. elegans embryo). The functional redundancy inferred from genetic analysis is due to biochemical redundancy of the two receptors, as GLP-1 can substitute for LIN-12 in cell fate decisions (Fitzgerald et al., 1993). lin-12 and glp-1 appear to have arisen by gene duplication (Yochem and Greenwald, 1989; Rudel and Kimble, 2002), so it is not surprising that some functions have been conserved.

As described below, genetic screens for other components of the LIN-12 and GLP-1 signaling pathways have exploited the highly penetrant phenotypic defects of single mutants in screens for extragenic suppressors and enhancers, as well as the Lag phenotype of the lin- $12 \mathrm{glp}$ - 1 double mutant. 


\section{Overview of signal transduction by LIN-12 and GLP-1}

LIN-12 and GLP-1 have all of the hallmarks that define the LIN-12/Notch family (Yochem and Greenwald, 1989; Yochem et al., 1988; Figure 1). They are type I transmembrane proteins that contain multiple EGF-like motifs commencing just after the signal sequence, followed by three copies of the LIN-12/Notch repeat (LNR) motif. A spacer region between the LNR motifs and the transmembrane domain contains two conserved cysteines. Vertebrate LIN-12/Notch proteins are cleaved by furin in this spacer region during their transit through the secretory system at "site 1" (Brou et al., 2000; Figure 1); it is not known whether the C. elegans proteins are also cleaved by furin, but Western blots of GLP-1 are consistent with this possibility (Crittenden et al., 1994). The intracellular domain of LIN-12/Notch proteins contains repeated "CDC10/Ankyrin" motifs, and a canonical PEST protein turnover motif.

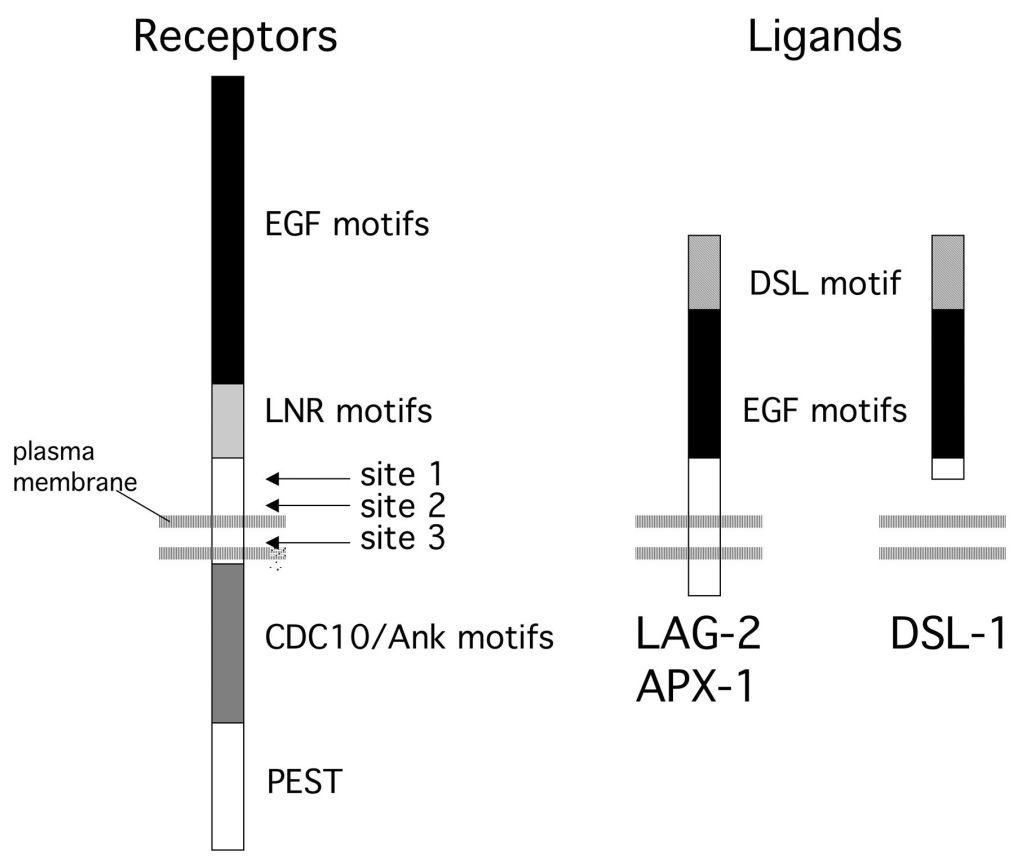

\section{LIN-12, GLP-1}

Figure 1. Schematic representation of . elegans LIN-12/Notch and DSL proteins. Conserved motifs: EGF = epidermal growth factor-like motif, LNR $=$ LIN-12/Notch repeat motif, CDC10/Ank = a motif found in yeast CDC10, SWI6, and Ankyrin. There are two C. elegans LIN-12/Notch proteins, LIN-12 and GLP-1; both have the same arrangement of motifs, as represented by the single cartoon. Inferred cleavage sites are shown (see also Figure 2). There are ten predicted DSL proteins; representative transmembrane and secreted DSL proteins are shown. All ten DSL proteins have a single amino-terminal DSL domain, followed by one or more EGF motifs.

Ligands for LIN-12 and GLP-1 (Figure 1) are members of the "DSL" family, an acronym derived from

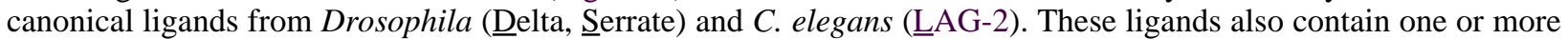
EGF motifs, as well as an amino terminal motif that is unique to the family, the "DSL motif". Binding of DSL ligands to LIN-12/Notch leads to shedding of the LIN-12/Notch ectodomain via cleavage at "site 2" within the spacer region of the extracellular domain (Figure 1 and Figure 2). The remaining transmembrane protein, with only a short extracellular stub, is cleaved constitutively within the transmembrane domain at "site 3" (Figure 1 and Figure 2), releasing the intracellular domain, which translocates to the nucleus (Kopan and Goate, 2000; Schroeter et al., 1998; Struhl and Adachi, 1998; Struhl and Adachi, 2000; Struhl et al., 1993). 


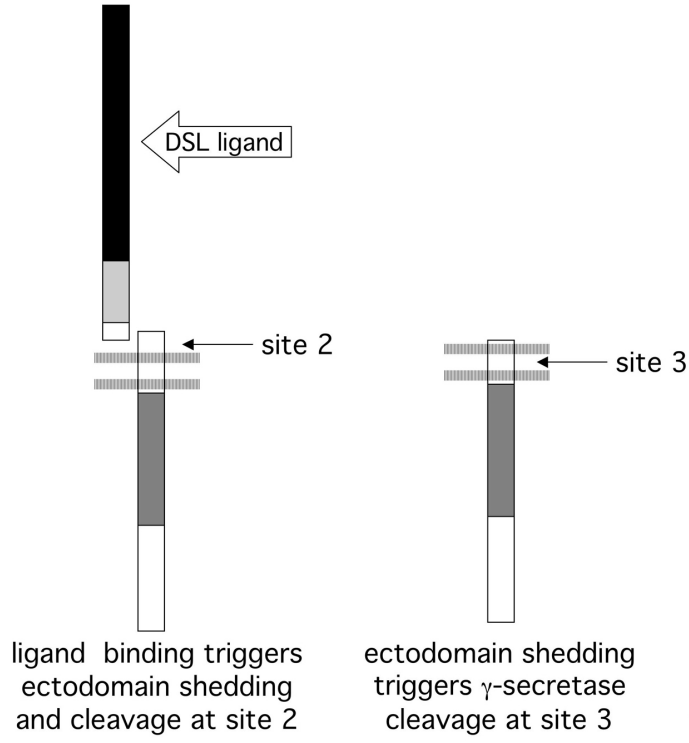

triggers $\gamma$-secretase

cleavage at site 3

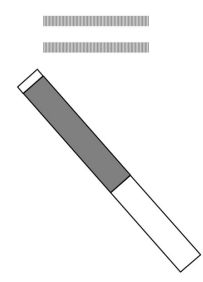

site 3 cleavage releases the intracellular domain for nuclear translocation

Figure 2. Mechanism of LIN-12/Notch signal transduction. See text for further description.

The intracellular domain contains a block of repeated CDC10/SWI6/Ankyrin motifs, which, along with the juxtamembrane region, mediate the physical interaction with the sequence specific DNA binding protein LAG-1 (Christensen et al., 1996; Roehl et al., 1996). LAG-1 is a member of the "CSL" family, an acronym derived from mammalian $\underline{\mathrm{CBF}} 1$ and Drosophila $\underline{\mathrm{S}} \mathrm{u}(\mathrm{H})$ as well as $\underline{\mathrm{LAG}}$-1. Studies of $\mathrm{Su}(\mathrm{H})$ and CBF1 suggest that the intracellular domain of LIN-12/Notch proteins convert CSL proteins from repressors into activators, thereby turning on the expression of target genes (Jarriault et al., 1995; Hsieh et al., 1996).

This mechanism of signal transduction was first suggested when expression of the intracellular domains of LIN-12 in C. elegans and Notch in Drosophila caused constitutive activation, with accumulation of the intracellular domain of Notch observed in the nucleus (Struhl et al.,1993). Then, it was found that the CSL DNA binding protein mediated transcription of Notch target genes and associated physically with the intracellular domain of Notch (Jarriault et al., 1995; Tamura et al., 1995). However, the intracellular domain could not be visualized in the nucleus under normal circumstances, and alternate models to explain these observations did not fall out of favor until compelling in vivo analysis in Drosophila (Struhl and Adachi, 1998) and biochemical analysis in mammalian cells (Schroeter et al., 1998) demonstrated ligand-dependent cleavage and nuclear translocation correlated with signal transduction.

\section{Identification and characterization of core components}

Given the mechanism of signal transduction, the core components of the pathway may be considered to be proteins that are directly involved in liberating the intracellular domain from its membrane tether, and components of the transcriptional activation complex that includes the intracellular domain. Genetic analysis in C. elegans has been important in identifying such components, particularly of the site 3 (transmembrane domain) protease complex. The core components and their Drosophila and mammalian counterparts are summarized in Table 1. In this section, I will detail how the core components were identified and characterized in C. elegans, and briefly summarize the state of understanding about these components from work in C. elegans and other systems.

\subsection{Ligands}

The first identified C. elegans ligand, lag-2, was recovered in two different genetic screens: null alleles of lag-2 were identified based on the Lag phenotype they confer (Lambie and Kimble, 1991), and unusual antimorphic alleles were identified as dominant suppressors of the egg-laying defect caused by a lin-12(d) mutation (see below; Tax and Thomas, 1994; Tax et al., 1997). Molecular cloning and sequence analysis revealed that lag-2 encodes a protein homologous to the known Drosophila Notch ligands Delta and Serrate (Henderson et al., 1994; Tax and Thomas, 1994). Another $C$. elegans ligand, apx-1, was identified in a genetic screen for maternal-effect lethal mutants (Mango et al., 1994; Mello et al., 1994). With the completion of the genomic sequence, it became clear that there are a total of ten genes that encode members of the DSL ligand family (Chen and Greenwald, 2004b). One of 
the new predicted ligands, DSL-1, was shown to be a secreted protein that is functionally redundant with lag-2 and apx-1 in LIN-12-mediated vulval precursor cell fate specification (Chen and Greenwald, 2004b; see also Vulval development).

\subsection{Site 2 (extracellular) cleavage}

sup-17 was identified as a suppressor of the 0 AC defect caused by lin-12(d) mutations (Tax et al., 1997) and found to encode a metalloprotease of the ADAM family (Wen et al., 1997). Its Drosophila homolog, Kuzbanian, was also implicated in Notch signaling (Pan and Rubin, 1997; Rooke et al., 1996; Sotillos et al., 1997). However, in mammals, a different ADAM protein, TACE, has been implicated in the site 2 cleavage (Brou et al., 2000). sup-17 is a positive factor for lin-12/Notch activity and appears to function cell autonomously, but there has not been a direct demonstration of its involvement in the site 2 cleavage. The $C$. elegans TACE ortholog, adm-4, also appears to be a positive factor for lin-12/Notch activity in some cell fate decisions; sup-17 and adm-4 are functionally redundant for at least a subset of LIN-12/Notch mediated decisions in C. elegans, but there may also be additional redundant proteases that can mediate the extracellular cleavage (Jarriault and Greenwald, 2005).

\subsection{Site 3 (transmembrane) cleavage}

The protein complex that mediates the site 3 cleavage of LIN-12/Notch also mediates the transmembrane cleavage of the $\beta$-amyloid precursor protein ( $\beta$-APP), and has been termed " $\gamma$-secretase" (Kopan and Goate, 2000). Four proteins are sufficient to reconstitute $\gamma$-secretase activity in yeast (Edbauer et al., 2003). Genetic analysis in $C$. elegans has been instrumental in identifying these components and in illuminating their roles.

The first evidence of a link between the LIN-12/Notch and $\beta$-APP transmembrane cleavage events came from the recovery of sel-12 as a suppressor of lin-12(d) (Levitan and Greenwald, 1995). sel-12 encodes a Presenilin, which had, at about the same time, been identified as a major genetic locus in familial early-onset Alzheimer's disease (Sherrington et al., 1995). A second C. elegans presenilin, hop-1, appears to be functionally redundant with sel-12, and genetic analysis of these genes in $C$. elegans established that Presenilin activity is critical for LIN-12/Notch signaling ( $\mathrm{Li}$ and Greenwald, 1997; Westlund et al., 1999). SEL-12/Presenilin is believed to be the catalytic subunit of the $\gamma$-secretase protease complex (Kopan and Goate, 2000). Analysis of SEL-12 revealed what is now generally accepted as the topology of Presenilin: eight membrane-spanning segments with a large cytosolic loop between the sixth and seventh transmembrane domains (Li and Greenwald, 1996, 1998; see Figure 3). These two transmembrane domains contain conserved aspartyl residues that appear to be the catalytic aspartates of the protease (Kopan and Goate, 2000).

Other genetic screens in $C$. elegans yielded the other three components of $\gamma$-secretase. Mutations that confer the anterior pharynx (Aph) defect resulting from failure of $g l p-1$ signaling at the 12 cell stage of embryogenesis yielded two genes, aph-1 and aph-2 (Goutte et al., 2000; Goutte et al., 2002). The ortholog of APH-2 is Nicastrin, which was also identified as a genetic locus in familial early-onset Alzheimer's disease (Yu et al., 2000). aph-1 as well as the fourth component of $\gamma$-secretase, pen-2, were identified in a screen for mutations that result in synthetic phenotypes when combined with loss of sel-12 or aph-2 activity (Francis et al., 2002) Biochemical analysis, principally in mammalian cell culture, has suggested that Nicastrin and Aph-1 form an initial complex, which binds to and stabilizes the Presenilin holoprotein; Pen-2 then associates with this tripartite complex to confer $\gamma$-secretase activity (reviewed in Iwatsubo, 2004). Endoproteolysis in the cytosolic loop of SEL-12/Presenilin (Li and Greenwald, 1996; Li and Greenwald, 1998; Thinakaran et al., 1996) is facilitated by Pen-2 and is believed to be essential for $\gamma$-secretase activity (reviewed in Iwatsubo, 2004), although deletion of this region does not appreciably abrogate Presenilin activity in C. elegans (Levitan et al., 1996). 


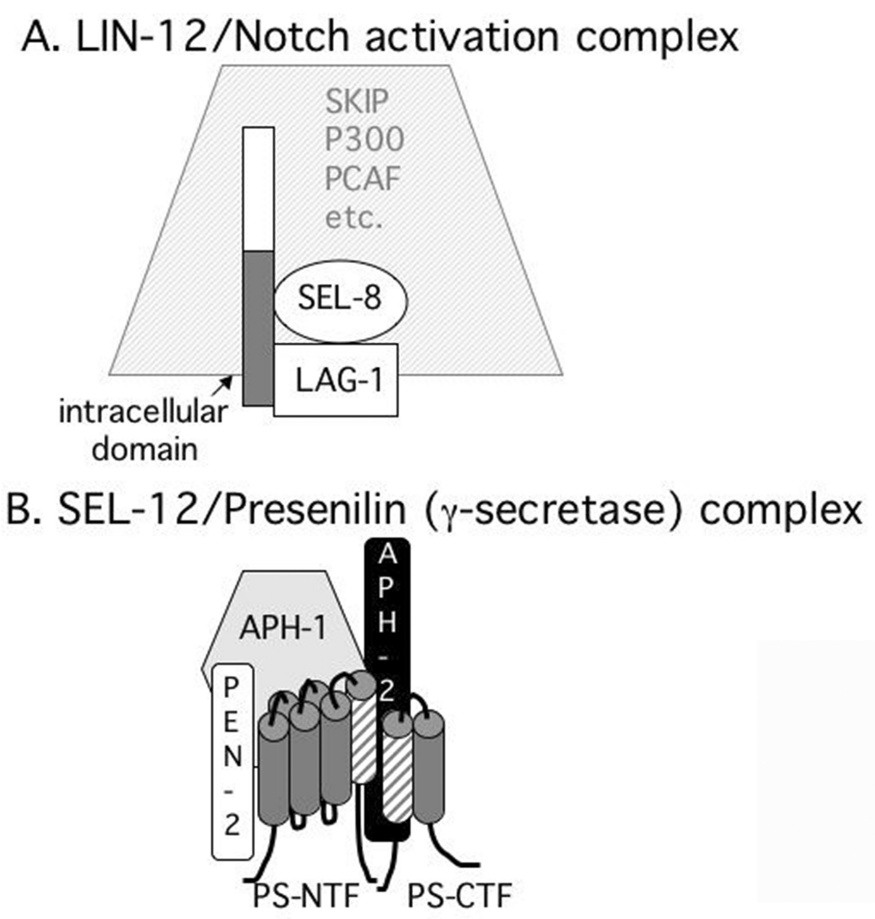

Figure 3. Core component complexes. A. The LIN-12/Notch activation complex. The intracellular domain of LIN-12 or GLP-1 forms a ternary complex with LAG-1 and SEL-8, which are both essential for lin-12 and glp-1 activity (Lambie and Kimble, 1991; Tax et al.,1997; Doyle et al., 2000; Petcherski and Kimble, 2000). B. The SEL-12/Presenilin ( $\gamma$-secretase) complex. SEL-12 and HOP-1, the C. elegans Presenilins (PS), adopt an eight transmembrane domain topology and are cleaved into N-terminal (PS NTF) and C-terminal (PS CTF) fragments. Transmembrane domains 6 and 7 contain catalytic aspartyl residues (represented by shading). The contributions of the two-pass transmembrane protein PEN-2, the seven-pass transmembrane protein APH-1, and the single-pass transmembrane protein APH-2/Nicastrin are described in the text.

Table 1. Core components of the LIN-12/Notch pathway

\begin{tabular}{|c|c|c|c|}
\hline Role & C. elegans & Drosophila & Mammals \\
\hline Ligand & $\begin{array}{l}\text { LAG-2, APX-1, } \\
\text { DSL-1, } 7 \text { others }\end{array}$ & Delta, Serrate & $\begin{array}{c}\text { Jagged, Delta-like, } \\
4 \text { others }\end{array}$ \\
\hline Receptor & LIN-12, GLP-1 & Notch & Notch 1-4 \\
\hline Site 2 cleavage ${ }^{\mathrm{a}}$ & $\begin{array}{l}\text { SUP-17 } \\
\text { ADM-4 }\end{array}$ & $\begin{array}{l}\text { Kuzbanian } \\
\text { (TACE) }\end{array}$ & $\begin{array}{l}\text { (ADAM-10) } \\
\text { TACE }\end{array}$ \\
\hline Site 3 cleavage & $\begin{array}{c}\text { SEL-12, HOP-1 } \\
\text { APH-1 } \\
\text { APH-2 } \\
\text { PEN-2 }\end{array}$ & $\begin{array}{l}\text { Presenilin } \\
\text { (Aph-1) } \\
\text { Nicastrin } \\
\text { (Pen-2) }\end{array}$ & $\begin{array}{c}\text { Presenilin 1, } 2 \\
\text { APH-1 } \\
\text { Nicastrin } \\
\text { PEN-2 }\end{array}$ \\
\hline Nuclear complex & $\begin{array}{l}\text { LAG-1 } \\
\text { SEL- }{ }^{b}\end{array}$ & $\begin{array}{c}\mathrm{Su}(\mathrm{H}) \\
\text { Mastermind }\end{array}$ & $\begin{array}{c}\text { CBF1/RBP-J } \\
\text { Mastermind-like }\end{array}$ \\
\hline
\end{tabular}

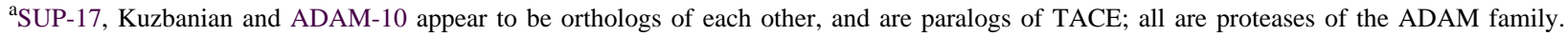
Orthology is defined by BLAST searching and phylogenetic analysis (e.g., as in Jarriault and Greenwald, 2005). ADM-4 and TACE also appear to be orthologs. There is evidence for functional redundancy of sup-17 and adm-4 (TACE) in C. elegans, although sup-17 appears to have a more significant role (Jarriault and Greenwald, 2005). In Drosophila the genetic evidence suggests that Kuzbanian alone may mediate S2 cleavage (Rooke et al., 1996; Pan and Rubin, 1997; Sottilos et al., 1997). We note that placement of SUP-17 at this step is consistent with available genetic information and with the Drosophila data. In mammals, TACE has been demonstrated to cleave Notch in biochemical assays (Brou et al., 2000), but genetic analysis is consistent with functionally redundant proteases and ADAM10 involvement.

${ }^{b}$ Official name, first published in Tax et al. (1997); also known as LAG-3. 


\subsection{The nuclear complex}

As mentioned above, CSL proteins are DNA binding proteins that are part of the transcriptional activation complex that forms with the LIN-12/Notch intracellular domain (see also Figure 3). Prior to LIN-12/Notch activation, CSL proteins have transcriptional repressor activity, and genetic analysis in Drosophila has established distinct roles for transcriptional repression by CSL proteins in development (e.g., Morel and Schweisguth, 2000). Association with the LIN-12/Notch intracellular domain is believed to lead to transcriptional activation in two steps: first by displacing corepressors, then by recruiting coactivators (Hsieh et al., 1996; Jarriault et al., 1995).

In C. elegans, the CSL protein is called LAG-1. Strong hypomorphic and null alleles of lag- 1 were recovered in a screen for mutations that cause a Lag phenotype, suggesting that lag- 1 is a critical component of LIN-12/Notch signaling (Lambie and Kimble, 1991). In addition, weaker alleles of lag-1 have also been recovered in a screen for alleles that strongly increase the severity of a weak $g l p-1$ loss-of-function phenotype in the germ line (Qiao et al., 1995) and in screens for suppression of the 0 AC defect caused by a lin-12(d) mutation (Katic et al., submitted). lag- 1 was cloned and shown to be orthologous to Drosophila $\mathrm{Su}(\mathrm{H})$ and mammalian CBF1/RBP-J, leading to the acronym CSL for this family of DNA binding proteins (Christensen et al., 1996) Indeed, LAG-1 appears to have the same binding specificity as its orthologs (Christensen et al., 1996). The crystal structure of LAG-1 complexed with target DNA revealed that the amino and carboxy-terminal domains are strikingly similar to a region oftranscription factors of the NF- $\mathrm{\kappa B} / \mathrm{Rel}$ family, with the insertion of an unusual beta-trefoil domain (Kovall and Hendrickson, 2004). The beta-trefoil domain appears to have roles in both the recognition of target DNA and in mediating mutually-exclusive interactions with either corepressors or the intracellular domain of LIN-12/Notch, providing a structural basis for the switch between repressor and activator forms (Kovall and Hendrickson, 2004).

The C. elegans transcription activation complex also is likely to contain SEL-8 (Figure 3). A single non-null allele of sel-8 was identified as a suppressor of the 0 AC defect caused by a lin-12(d) mutation (Tax et al., 1997). sel-8 encodes a novel, glutamine rich, nuclear protein (Doyle et al., 2000). SEL-8 was also identified based on the formation of a ternary complex with LAG-1 and the intracellular domain of GLP-1 in yeast; it was referred to as LAG-3 in this study (Petcherski and Kimble, 2000a). Based on fact that both SEL-8 and Mastermind have glutamine-rich stretches, and the observation that both form ternary complexes with the intracellular domains of LIN-12/Notch and CSL proteins, Petcherski and Kimble (2000b) proposed that SEL-8 serves the same function as Drosophila and mammalian Mastermind. Mastermind has been well-characterized as an integral part of the complex that binds DNA (Kitagawa et al., 2001; Wu et al., 2000). In addition to contributing to transcriptional activation of target genes, Mastermind may enhance phosphorylation and turnover of the Notch intracellular domain (Fryer et al., 2002).

At this time, these three components--the intracellular domain of LIN-12/Notch, a CSL protein, and SEL-8/Mastermind--are believed to be the core of the transcriptional activation complex formed when LIN-12/Notch is activated (Figure 3; Fryer et al., 2002). Numerous other proteins, which play more general roles in transcriptional activation, are recruited through interactions with this core complex. Among the proteins recruited are $\mathrm{CBP} / \mathrm{p} 300$, which is recruited by association with Mastermind, and the p300-associated protein PCAF (Fryer et al., 2002), and SKIP, which physically associates with both CBF1 and the Notch intracellular domain (Zhou et al., 2000).

\section{Identification of modulators of $/ i n-12$ and $g / p-1$ activity}

Various genetic screens have identified many modulators of $l i n-12$ and $g l p-1$ activity. For the purposes of this discussion, I consider a modulator to be any gene whose activity influences lin- 12 and/or $g l p-1$ activity, but whose gene product has not been established to be a core component. In some cases, new information may at some future date lead to reclassification.

\subsection{Suppressors of lin-12(d)}

In lin-12(d) mutants, excess lin-12 activity alters a cell fate decision during gonadogenesis (see below), leading to the absence of an $\mathrm{AC}$, and consequently to an inability to lay eggs. Extensive, but not saturated, screens for extragenic suppressors of this egg-laying defect have been performed (Greenwald et al., 1983; Tax et al., 1997; Katic et al., submitted). Most genes recovered in these screens, and in the screen for suppressors of a lin-12 hypomorph (see below), are named "sel" (uppressor/enhancer of lin-12), because their genetic behavior as a suppressor or enhancer depends on the nature of the lin-12 allele with which they are combined. Many of the sel genes also interact genetically with $g l p-1$. 
In addition to alleles of the core component genes lag-2, sel-8, sup-17 and lag-1, the screen for lin-12(d) suppressors has yielded mutations in genes for SEL-5, a serine/threonine kinase (Fares and Greenwald, 1999), SEL-7, a novel nuclear protein (Chen et al., 2004a), and SEL-6, a AAA-ATPase (Katic and Greenwald, in preparation; Tables 2 and 3). This screen has also yielded a loss-of-function allele of bre-5, which encodes a carbohydrate modifying enzyme that is homologous to Drosophila Brainiac (Griffitts et al., 2001), and several additional, as yet uncloned genes (Katic et al., submitted).

Table 2. Modulators of lin-12 and glp-1 activity. Genes listed here have displayed a genetic interaction with both $l i n-12$ and $g l p-1$ in at least one cell fate decision.

\begin{tabular}{|c|c|c|c|}
\hline Role & Inferred regulatory role & C. elegans & Mammals $^{\circ}$ \\
\cline { 2 - 4 } & + & SEL-7 & $?^{\mathrm{a}}$ \\
\cline { 2 - 4 } & & SPR-1 & CoREST \\
Nuclear/ & - & SPR-2 & Set/Nap \\
target genes & & SPR-3 & $?$ \\
& & SPR-4 & p110b \\
\hline ERAD/ & - & SPR-5 & Sel-1 \\
quality control & & SEL-1 & p24 \\
\hline Stability & - & SEL-9 & Sel-10/Fbw7 \\
\hline
\end{tabular}

a $?=$ no apparent mammalian ortholog based on amino acid sequence identity, but proteins divergent in sequence may perform equivalent functions. SPR-3 and SPR-4 contain zinc fingers (Lakowski et al., 2003). SEL-7 contains no recognizable amino acid sequence motifs and has only nematode orthologs.

\subsection{Suppressors of a lin-12 hypomorph}

Reversion of the egg-laying defect caused by partial loss of lin-12 activity yielded five genes (Sundaram and Greenwald, 1993). Three have been characterized in detail (Table 2). sel-1 encodes a protein that is involved in endoplasmic reticulum-associated protein degradation (Grant and Greenwald, 1996; Grant and Greenwald, 1997; Urano et al., 2002). sel-9 encodes a p24 protein involved in quality control of membrane protein trafficking (Wen and Greenwald, 1999). sel-10 encodes an E3 ubiquitin ligase that forms a complex with the intracellular domain of LIN-12 (Hubbard et al., 1997). Studies of mammalian orthologs have demonstrated that SEL-10 promotes the ubiquitin-mediated turnover of the intracellular domain of LIN-12/Notch proteins via phosphorylation of the PEST domain (Gupta-Rossi et al., 2001; Oberg et al., 2001; Wu et al., 2001).

\subsection{Suppressors of $g / p-1$ hypomorph}

Screens for suppressors of the germline defect caused by loss of zygotic $g l p-1$ activity (Maine and Kimble, 1993) or of the embryonic lethality caused by loss of maternal $g l p-1$ activity (A.M. Howell and J. Priess, personal communication) identified ten $\operatorname{sog}$ genes, for suppressor of $g l p-1$. None of these have been molecularly characterized. Other suppressors of $g l p-l$ sterility proved to be genes required for body morphology, which may indicate that the extracellular matrix influences $g l p-1$ activity in the germ line (Maine and Kimble, 1993).

\subsection{Enhancers of a glp-1 hypomorph}

Partial loss of $g l p-1$ activity provides a sensitized background to search for genes that promote $g l p-1$ activity, as reducing the activity of such genes will cause a synthetic Glp sterile phenotype. This strategy was used to screen for enhancers of the partial germline proliferation defect of a weak $g l p-l$ loss-of-function mutation and yielded five ego genes, for enhancers of $g l p-1$ (ㅁe), as well as weak alleles of lag-l (Qiao et al., 1995; Smardon et al., 2000). ego-1 plays multiple roles in germline development, and encodes an RNA-directed RNA polymerase (Smardon et al., 2000; Table 2). ego-1 mutants are resistant to RNAi, suggesting a link between germline development and cellular processes involved in post-transcriptional silencing (Smardon et al., 2000). ego-l also promotes heterochromatin assembly, and therefore may influence gene expression at the transcriptional level (E. Maine and 
W. Kelly, personal communication). Recent analysis of ego-5 suggests that regulation of DNA replication proteins may be part of the switch from mitotic to meiotic development (E. Maine, personal communication). The gene atx-2 was also identified by RNAi as an ego gene and found to be required for germline proliferation (Ciosk et al., 2004; Maine et al., 2004). atx-2 acts independently of GLP-1 signaling and in translational regulation in the germline (Ciosk et al., 2004; Maine et al., 2004).

\subsection{Enhancers of glp-1 gain-of-function}

Strong activation of $g l p-1$ in the germ line results in continued mitotic proliferation without meiosis, called the "tumorous" phenotype. A screen for enhancers of a weak tumorous phenotype caused by mild $g l p$ - 1 gain-of-function has identified tumorous enhancers of $g l p-1$ ( $t e g$ genes), potential negative regulators of $g l p-1$ activity in the germ line (D. Hansen and T. Schedl, personal communication). The teg genes are currently being analyzed in detail.

\subsection{Suppressors of Presenilin}

sel-12 null alleles are unable to lay eggs because of a defect in lin-12-mediated $\pi$ cell specification (Cinar et al., 2001). sel-12 null mutants are viable because they retain activity of a second presenilin gene, hop-1 (Li and Greenwald, 1997). Suppressors of the egg-laying defective phenotype of sel-12 mutants identified spr genes (suppressors of presenilin; Wen et al., 2000), four of which (SPR-1, SPR-3, SPR-4 and SPR-5; Table 2) appear to be members of a CoREST-containing corepressor complex (Eimer et al., 2002; Jarriault and Greenwald, 2002; Lakowski et al., 2003). The CoREST complex may be involved in the balance of repression and activation of lin-12 target genes or in regulating expression of a limiting component required for lin-12 activity.

sel-10 also suppresses the egg-laying defect of sel-12 mutants, and SEL-10 forms a complex with SEL-12, suggesting that sel-10 may regulate lin-12 activity through regulating SEL-12 as well LIN-12 levels (Wu et al., 1998).

Table 3. Modulators of lin-12 or glp-1 activity. Genes listed here have displayed a genetic interaction with either lin-12 or glp-1. All possible interactions have not necessarily been tested.

\begin{tabular}{|c|c|c|c|}
\hline Modulator of: & $\begin{array}{c}\text { Inferred regulatory } \\
\text { role }\end{array}$ & C. elegans & Mammals \\
\hline$g l p-1$ & + & EGO-1 & $?^{\text {a }}$ \\
\hline $\operatorname{lin}-12$ & - & SEL-2 & Neurobeachin \\
\hline $\operatorname{lin}-12$ & + & SEL-5 & AAK1 \\
\hline $\operatorname{lin}-12$ & + & BRE-5 & Brainiac glycosyltransferase \\
\hline
\end{tabular}

${ }^{\mathrm{a}} ?=$ no apparent ortholog based on amino acid sequence identity, but proteins divergent in sequence may perform equivalent functions.

\section{Lateral specification and induction mediated by LIN-12 and GLP-1}

LIN-12 and GLP-1 mediate many different cell-cell interactions during C. elegans development. Some of these interactions are considered elsewhere in WormBook (see Notch signaling in the C. elegans embryo, Vulval development, Male development, Hermaphrodite cell-fate specification, and Germline proliferation and its control) and so have not been catalogued here. The two general categories of cell-cell interactions are lateral specification and induction. Lateral specification (lateral inhibition) occurs when a group of equivalent cells interact among themselves to specify alternative cell fates. Induction occurs when equivalent cells choose alternative fates in response to an external source of ligand, with cells close to the source selecting one fate, and cells further away selecting another.

LIN-12/Notch proteins appear to be unique mediators of lateral specification during animal development. The archetypal example of lateral specification in C. elegans involves a cell fate decision between the anchor cell (AC) and ventral uterine precursor cell (VU) fates in early gonadogenesis in hermaphrodites. During the "AC/VU decision", two equivalent cells each have the potential to become an AC or a VU. The two cells interact with each other, via LAG-2 and LIN-12, so that only one becomes the AC. A key concept of general significance that emerged from studies of this decision is the importance of a feedback mechanism that drives the cells to adopt different fates: both Z1.ppp and Z4.aaa initially express both lin-12 and lag-2, but a small initial difference in lin-12 activity is 
amplified by reciprocal feedback loops in the presumptive AC and presumptive VU (Seydoux and Greenwald, 1989; Wilkinson et al., 1994). In the presumptive VU, LIN-12 activation by LAG-2 positively autoregulates lin-12 transcription and downregulates lag-2 transcription (Wilkinson et al., 1994). The latter step appears to involve post-transcriptional downregulation of HLH-2, a transcription factor required for lag-2 transcription, in response to LIN-12 activation (Karp and Greenwald, 2003).

LIN-12/Notch proteins, like many other receptor systems, also mediate inductive interactions during development. In these cases, receptor activity is essentially regulated by where the ligand is expressed or activated. The roles of $g l p-1$ in germline development (see Specification of the germ line) and pharyngeal induction are canonical inductive interactions: ligands produced in the distal tip cell of the somatic gonad activate GLP-1 in the germ line (Henderson et al., 1994), and ligands produced in one embryonic founder cell activate GLP-1 in descendants of another (Mickey et al., 1996; Shelton and Bowerman, 1996). However, even when ligand originates externally, there appear to be feedback mechanisms that affect receptor expression or activity. During germline development, there appears to be post-transcriptional up-regulation of GLP-1 accumulation (Berry et al., 1997). Another intriguing example is afforded by the lin-12-mediated generation of morphological twist of the intestinal tube: twist is initiated when the cells in the left half of the intestinal primordium contact external, LAG-2-expressing cells. and activation of LIN-12 by LAG-2 leads to downregulation of lin-12 transcription (Hermann etal., 2000).

Regulatory mechanisms integrate LIN-12/Notch signaling with other signaling events during animal development. For example, in $C$. elegans, cross-regulatory interactions between the LET-23/EGF receptor-Ras-MAP kinase pathway and LIN-12 ensure that the central three vulval precursor cells (VPCs) adopt the correct pattern of fates, represented as $2^{\circ}-1^{\circ}-2^{\circ}$ (see Vulval development). In the presumptive $1^{\circ} \mathrm{VPC}$, activation of LET-23 leads to transcription of DSL ligand genes (Chen and Greenwald, 2004b). However, in the presumptive $1^{\circ}$ VPC, ligand expression per se is not sufficient to activate LIN-12 in the flanking VPCs; in addition, endocytosis-mediated downregulation of LIN-12, also in response to LET-23 activation, must occur for the DSL ligands to be active (Shaye and Greenwald, 2002). In the presumptive $2^{\circ}$ VPCs, activation of LIN-12 leads to transcription of target genes that encode negative regulators of the EGF receptor-Ras-MAP kinase pathway, effectively squelching input from the inductive signaling pathway (Berset et al., 2001; Yoo et al., 2004).

\section{Future prospects}

With the core signal transduction mechanism largely elucidated, the emphasis has been shifting to understanding how signaling is modulated. One aspect is understanding how signaling is modulated in response to LIN-12/Notch activation itself, i.e. feedback mechanisms. There are likely to be many ways in which feedback modulation is achieved. Transcriptional regulation may involve different transcriptional regulatory circuits (see Notch signaling in the $C$. elegans embryo). Modulation is likely to involve different mechanisms for post-transcriptional regulation as well. Another aspect is understanding how LIN-12/Notch signaling is influenced by, and integrated with, other signaling inputs. This aspect, too, is likely to involve modulation of signaling by transcriptional and post-transcriptional mechanisms. Another important question for the future is how activation of LIN-12/Notch specifies different cell fate choices. One approach to answering this question is the identification and characterization of target genes--and one fruitful approach to identifying target genes is computational analysis to identify genes that contain potential LAG-1 binding sites in regulatory regions (Yoo et al., 2004).

\section{Acknowledgements}

Thanks to Marty Chalfie, Lisa Girard, Iskra Katic, Bob Johnston, Eleanor Maine, Sophie Jarriault, Jim Priess, Tim Schedl, and Gary Struhl for helpful comments on the manuscript. Work on the identification of genes that influence lin-12/Notch activity in my lab is supported by NIH CA095389. I am an Investigator of the Howard Hughes Medical Institute.

\section{References}

Austin, J., and Kimble, J. (1987). glp-1 is required in the germ line for regulation of the decision between mitosis and meiosis in C. elegans. Cell 51, 589-599. Abstract Article

Berry, L.W., Westlund, B., and Schedl, T. (1997). Germ-line tumor formation caused by activation of $g l p-1$, a Caenorhabditis elegans member of the Notch family of receptors. Development 124, 925-936. Abstract 
Berset, T., Hoier, E.F., Battu, G., Canevascini, S., and Hajnal, A. (2001). Notch inhibition of RAS signaling through MAP kinase phosphatase LIP-1 during C. elegans vulval development. Science 291, 1055-1058. Abstract Article

Brou, C., Logeat, F., Gupta, N., Bessia, C., LeBail, O., Doedens, J.R., Cumano, A., Roux, P., Black, R.A., and Israel, A. (2000). A novel proteolytic cleavage involved in Notch signaling: the role of the disintegrin-metalloprotease TACE. Mol. Cell 5, 207-216. Abstract Article

Chao, M.Y., Larkins-Ford, J., Tucey, T.M., and Hart, A.C. (2005). lin-12 Notch functions in the adult nervous system of C. elegans. BMC Neurosci. 6, 45. Article

Chen, J., Li, X., and Greenwald, I. (2004a). sel-7, a positive regulator of lin-12 activity, encodes a novel nuclear protein in Caenorhabditis elegans. Genetics 166, 151-160. Article

Chen, N., and Greenwald, I. (2004b). The lateral signal for LIN-12/Notch in C. elegans vulval development comprises redundant secreted and transmembrane DSL proteins. Dev. Cell 6, 183-192. Abstract Article

Christensen, S., Kodoyianni, V., Bosenberg, M., Friedman, L., and Kimble, J. (1996). lag-1, a gene required for lin-12 and glp-1 signaling in Caenorhabditis elegans, is homologous to human CBF1 and Drosophila $\mathrm{Su}(\mathrm{H})$. Development 122, 1373-1383. Abstract

Cinar, H.N., Sweet, K.L., Hosemann, K.E., Earley, K., and Newman, A.P. (2001). The SEL-12 presenilin mediates induction of the Caenorhabditis elegans uterine pi cell fate. Dev. Biol. 237, 173-182. Abstract Article

Ciosk, R., DePalma, M., and Priess, J.R. (2004). ATX-2, the C. elegans ortholog of ataxin 2, functions in translational regulation in the germline. Development 131, 4831-4841. Article

Crittenden, S.L., Troemel, E.R., Evans, T.C., and Kimble, J. (1994). GLP-1 is localized to the mitotic region of the C. elegans germ line. Development 120, 2901-2911. Abstract

Doyle, T.G., Wen, C., and Greenwald, I. (2000). SEL-8, a nuclear protein required for LIN-12 and GLP-1 signaling in Caenorhabditis elegans. Proc. Natl. Acad. Sci. USA 97, 7877-7881. Abstract Article

Edbauer, D., Winkler, E., Regula, J.T., Pesold, B., Steiner, H., and Haass, C. (2003). Reconstitution of $\gamma$-secretase activity. Nat. Cell Biol. 5, 486-488. Abstract Article

Eimer, S., Donhauser, R., and Baumeister, R. (2002). The Caenorhabditis elegans presenilin sel-12 is required for mesodermal patterning and muscle function. Dev. Biol. 251, 178-192. Article

Eimer, S., Lakowski, B., Donhauser, R., and Baumeister, R. (2002). Loss of spr-5 bypasses the requirement for the C.elegans presenilin sel-12 by derepressing hop-1. EMBO J. 21, 5787-5796. Abstract Article

Fares, H., and Greenwald, I. (1999). SEL-5, a serine/threonine kinase that facilitates lin-12 activity in Caenorhabditis elegans. Genetics 153, 1641-1654.

Ferguson, E.L., and Horvitz, H.R. (1985). Identification and characterization of 22 genes that affect the vulval cell lineages of the nematode Caenorhabditis elegans. Genetics 110,17-72. Abstract

Fitzgerald, K., Wilkinson, H.A., and Greenwald, I. (1993). glp-1 can substitute for lin-12 in specifying cell fate decisions in Caenorhabditis elegans. Development 119, 1019-1027. Abstract

Francis, R., McGrath, G., Zhang, J., Ruddy, D. A., Sym, M., Apfeld, J., Nicoll, M., Maxwell, M., Hai, B., Ellis, M.C., et al. (2002). aph-1 and pen-2 are required for Notch pathway signaling, gamma-secretase cleavage of $\beta$ APP, and presenilin protein accumulation. Dev. Cell 3, 85-97. Abstract Article

Goutte, C., Hepler, W., Mickey, K.M., and Priess, J.R. (2000). aph-2 encodes a novel extracellular protein required for GLP-1-mediated signaling. Development 127, 2481-2492. Abstract 
Goutte, C., Tsunozaki, M., Hale, V.A., and Priess, J.R. (2002). APH-1 is a multipass membrane protein essential for the Notch signaling pathway in Caenorhabditis elegans embryos. Proc. Natl. Acad. Sci. USA 99, 775-779. Abstract Article

Grant, B., and Greenwald, I. (1996). The Caenorhabditis elegans sel-1 gene, a negative regulator of lin-12 and glp-1, encodes a predicted extracellular protein. Genetics 143, 237-247.

Grant, B., and Greenwald, I. (1997). Structure, function, and expression of SEL-1, a negative regulator of LIN-12 and GLP-1 in C. elegans. Development 124, 637-644.

Greenwald, I. (1985). lin-12, a nematode homeotic gene, is homologous to a set of mammalian proteins that includes epidermal growth factor. Cell 43, 583-590. Abstract Article

Greenwald, I.S., Sternberg, P.W., and Horvitz, H.R. (1983). The lin-12 locus specifies cell fates in Caenorhabditis elegans. Cell 34, 435-444. Abstract Article

Gridley, T. (2003). Notch signaling and inherited disease syndromes. Hum. Mol. Genet. 12 Spec No 1, R9-R13. Abstract Article

Griffitts, J.S., Whitacre, J.L., Stevens, D. E., and Aroian, R.V. (2001). Bt toxin resistance from loss of a putative carbohydrate-modifying enzyme. Science 293, 860-864. Abstract Article

Gupta-Rossi, N., Le Bail, O., Gonen, H., Brou, C., Logeat, F., Six, E., Ciechanover, A., and Israel, A. (2001). Functional interaction between SEL-10, an F-box protein, and the nuclear form of activated Notch1 receptor. J. Biol. Chem. 276, 34371-34378. Abstract Article

Hansson, E.M., Lendahl, U., and Chapman, G. (2004). Notch signaling in development and disease. Semin. Cancer Biol. 14, 320-328. Abstract Article

Harper, J.A., Yuan, J.S., Tan, J.B., Visan, I., and Guidos, C.J. (2003). Notch signaling in development and disease. Clin. Genet. 64, 461-472. Abstract Article

Henderson, S.T., Gao, D., Lambie, E.J., and Kimble, J. (1994). lag-2 may encode a signaling ligand for the GLP-1 and LIN-12 receptors of C. elegans. Development 120, 2913-2924. Abstract

Hermann, G.J., Leung, B., and Priess, J.R. (2000). Left-right asymmetry in C. elegans intestine organogenesis involves a LIN-12/Notch signaling pathway. Development 127, 3429-3440. Abstract

Hsieh, J.J., Henkel, T., Salmon, P., Robey, E., Peterson, M.G., and Hayward, S.D. (1996). Truncated mammalian Notch1 activates CBF1/RBPJk-repressed genes by a mechanism resembling that of Epstein-Barr virus EBNA2. Mol. Cell Biol. 16, 952-959. Abstract

Hubbard, E.J., Wu, G., Kitajewski, J., and Greenwald, I. (1997). sel-10, a negative regulator of lin-12 activity in Caenorhabditis elegans, encodes a member of the CDC4 family of proteins. Genes Dev. 11, 3182-3193.

Hutter, H., and Schnabel, R. (1994). glp-1 and inductions establishing embryonic axes in C. elegans. Development 120, 2051-2064. Abstract

Jarriault, S., Brou, C., Logeat, F., Schroeter, E.H., Kopan, R., and Israel, A. (1995). Signalling downstream of activated mammalian Notch. Nature 377, 355-358. Abstract Article

Jarriault, S., and Greenwald, I. (2005). Evidence for functional redundancy between C. elegans ADAM proteins SUP-17/Kuzbanian and ADM-4/TACE. Dev. Biol. 287, 1-10. Abstract Article

Jarriault, S., and Greenwald, I. (2002). Suppressors of the egg-laying defective phenotype of sel-12 presenilin mutants implicate the CoREST corepressor complex in LIN-12/Notch signaling in C. elegans. Genes Dev. 16, 2713-2728. Abstract Article 
Karp, X., and Greenwald, I. (2003). Post-transcriptional regulation of the E/Daughterless ortholog HLH-2, negative feedback, and birth order bias during the AC/VU decision in C. elegans. Genes Dev. 17, 3100-3111. Abstract Article

Kidd, S., Kelley, M.R., and Young, M.W. (1986). Sequence of the notch locus of Drosophila melanogaster: relationship of the encoded protein to mammalian clotting and growth factors. Mol. Cell Biol. 6, 3094-3108. Abstract

Kitagawa, M., Oyama, T., Kawashima, T., Yedvobnick, B., Kumar, A., Matsuno, K., and Harigaya, K. (2001). A human protein with sequence similarity to Drosophila mastermind coordinates the nuclear form of notch and a CSL protein to build a transcriptional activator complex on target promoters. Mol. Cell Biol. 21, 4337-4346. Abstract Article

Kopan, R., and Goate, A. (2000). A common enzyme connects notch signaling and Alzheimer's disease. Genes Dev. 14, 2799-2806. Abstract Article

Kovall, R.A., and Hendrickson, W.A. (2004). Crystal structure of the nuclear effector of Notch signaling, CSL, bound to DNA. EMBO J. 23, 3441-3451. Abstract Article

Lakowski, B., Eimer, S., Gobel, C., Bottcher, A., Wagler, B., and Baumeister, R. (2003). Two suppressors of sel-12 encode $\mathrm{C} 2 \mathrm{H} 2$ zinc-finger proteins that regulate presenilin transcription in Caenorhabditis elegans. Development 130, 2117-2128. Article

Lambie, E.J., and Kimble, J. (1991). Two homologous regulatory genes, lin-12 and glp-1, have overlapping functions. Development 112, 231-240. Abstract

Laughon, A., and Scott, M.P. (1984). Sequence of a Drosophila segmentation gene: protein structure homology with DNA-binding proteins. Nature 310, 25-31. Article

Levitan, D., Doyle, T.G., Brousseau, D., Lee, M.K., Thinakaran, G., Slunt, H.H., Sisodia, S.S., and Greenwald, I. (1996). Assessment of normal and mutant human presenilin function in Caenorhabditis elegans. Proc. Natl. Acad. Sci. USA 93, 14940-14944. Article

Levitan, D., and Greenwald, I. (1995). Facilitation of lin-12-mediated signalling by sel-12, a Caenorhabditis elegans S182 Alzheimer's disease gene. Nature 377, 351-354. Abstract Article

Li, X., and Greenwald, I. (1996). Membrane topology of the Caenorhabditis elegans SEL-12 presenilin. Neuron 17, 1015-1021. Abstract Article

Li, X., and Greenwald, I. (1997). HOP-1, a Caenorhabditis elegans presenilin, appears to be functionally redundant with SEL-12 presenilin and to facilitate LIN-12 and GLP-1 signaling. Proc. Natl. Acad. Sci. USA 94, 12204-12209. Abstract Article

Li, X., and Greenwald, I. (1998). Additional evidence for an eight-transmembrane-domain topology for Caenorhabditis elegans and human presenilins. Proc. Natl. Acad. Sci. USA 95, 7109-7114. Abstract Article

Maine, E.M., and Kimble, J. (1993). Suppressors of $g l p-1$, a gene required for cell communication during development in Caenorhabditis elegans, define a set of interacting genes. Genetics 135, 1011-1022.

Mango, S.E., Thorpe, C.J., Martin, P.R., Chamberlain, S.H., and Bowerman, B. (1994). Two maternal genes, apx-1 and pie-1, are required to distinguish the fates of equivalent blastomeres in the early Caenorhabditis elegans embryo. Development 120, 2305-2315. Abstract

McGinnis, W., Levine, M.S., Hafen, E., Kuroiwa, A., and Gehring, W.J. (1984). A conserved DNA sequence in homoeotic genes of the Drosophila Antennapedia and bithorax complexes. Nature 308, 428-433. Article

Mello, C.C., Draper, B.W., and Priess, J.R. (1994). The maternal genes apx-1 and glp-1 and establishment of dorsal-ventral polarity in the early C. elegans embryo. Cell 77, 95-106. Abstract Article 
Mickey, K.M., Mello, C.C., Montgomery, M.K., Fire, A., and Priess, J.R. (1996). An inductive interaction in 4-cell stage C. elegans embryos involves APX-1 expression in the signalling cell. Development 122, 1791-1798.

Morel, V., and Schweisguth, F. (2000). Repression by suppressor of hairless and activation by Notch are required to define a single row of single-minded expressing cells in the Drosophila embryo. Genes Dev. 14, 377-388. Abstract

Moskowitz, I.P., Gendreau, S.B., and Rothman, J.H. (1994). Combinatorial specification of blastomere identity by glp-1-dependent cellular interactions in the nematode Caenorhabditis elegans. Development 120, 3325-3338. Abstract

Newman, A.P., White, J.G., and Sternberg, P.W. (1995). The Caenorhabditis elegans lin-12 gene mediates induction of ventral uterine specialization by the anchor cell. Development 121,263-271. Abstract

Oberg, C., Li, J., Pauley, A., Wolf, E., Gurney, M., and Lendahl, U. (2001). The Notch intracellular domain is ubiquitinated and negatively regulated by the mammalian Sel-10 homolog. J. Biol. Chem. 276, 35847-35853. Abstract Article

Pan, D., and Rubin, G.M. (1997). Kuzbanian controls proteolytic processing of Notch and mediates lateral inhibition during Drosophila and vertebrate neurogenesis. Cell 90, 271-280. Abstract Article

Pepper, A.S., Killian, D.J., and Hubbard, E.J. (2003). Genetic analysis of Caenorhabditis elegans glp-1 mutants suggests receptor interaction or competition. Genetics 163, 115-132. Abstract

Petcherski, A.G., and Kimble, J. (2000a). LAG-3 is a putative transcriptional activator in the C. elegans Notch pathway. Nature 405, 364-368. Abstract Article

Petcherski, A.G., and Kimble, J. (2000b). Mastermind is a putative activator for Notch. Curr. Biol. 10, R471-R473. Article

Priess, J.R., Schnabel, H., and Schnabel, R. (1987). The glp-1 locus and cellular interactions in early C. elegans embryos. Cell 51,601-611. Abstract Article

Qiao, L., Lissemore, J.L., Shu, P., Smardon, A., Gelber, M.B., and Maine, E.M. (1995). Enhancers of glp-1, a gene required for cell-signaling in Caenorhabditis elegans, define a set of genes required for germline development. Genetics 141, 551-569.

Roehl, H., Bosenberg, M., Blelloch, R., and Kimble, J. (1996). Roles of the RAM and ANK domains in signaling by the C. elegans GLP-1 receptor. EMBO J. 15, 7002-7012. Abstract

Rooke, J., Pan, D., Xu, T., and Rubin, G.M. (1996). KUZ, a conserved metalloprotease-disintegrin protein with two roles in Drosophila neurogenesis. Science 273, 1227-1231. Abstract

Schroeter, E.H., Kisslinger, J.A., and Kopan, R. (1998). Notch-1 signalling requires ligand-induced proteolytic release of intracellular domain. Nature 393,382-386. Abstract Article

Seydoux, G., and Greenwald, I. (1989). Cell autonomy of lin-12 function in a cell fate decision in C. elegans. Cell 57, 1237-1245. Article

Shaye, D.D., and Greenwald, I. (2002). Endocytosis-mediated downregulation of LIN-12/Notch upon Ras activation in Caenorhabditis elegans. Nature 420, 686-690. Abstract Article

Shelton, C.A., and Bowerman, B. (1996). Time-dependent responses to $g l p-1$-mediated inductions in early $C$. elegans embryos. Development 122, 2043-2050.

Sherrington, R., Rogaev, E.I., Liang, Y., Rogaeva, E.A., Levesque, G., Ikeda, M., Chi, H., Lin, C., Li, G., and Holman, K. (1995). Cloning of a gene bearing missense mutations in early-onset familial Alzheimer's disease. Nature 375, 754-760. Abstract Article 
Smardon, A., Spoerke, J.M., Stacey, S.C., Klein, M.E., Mackin, N., and Maine, E.M. (2000). EGO-1 is related to RNA-directed RNA polymerase and functions in germ-line development and RNA interference in C. elegans. Curr. Biol. 10, 169-178. Article

Sotillos, S., Roch, F., and Campuzano, S. (1997). The metalloprotease-disintegrin Kuzbanian participates in Notch activation during growth and patterning of Drosophila imaginal discs. Development 124, 4769-4779. Abstract

Struhl, G., and Adachi, A. (1998). Nuclear access and action of notch in vivo. Cell 93, 649-660. Abstract Article

Struhl, G., and Adachi, A. (2000). Requirements for presenilin-dependent cleavage of notch and other transmembrane proteins. Mol. Cell 6, 625-636. Abstract Article

Struhl, G., Fitzgerald, K., and Greenwald, I. (1993). Intrinsic activity of the Lin-12 and Notch intracellular domains in vivo. Cell 74, 331-345. Abstract Article

Sundaram, M., and Greenwald, I. (1993). Suppressors of a lin-12 hypomorph define genes that interact with both lin-12 and glp-1 in Caenorhabditis elegans. Genetics 135, 765-783.

Tamura, K., Taniguchi, Y., Minoguchi, S., Sakai, T., Tun, T., Furukawa, T., and Honjo, T. (1995). Physical interaction between a novel domain of the receptor Notch and the transcription factor RBP-J. kappa/Su(H). Curr. Biol. 5, 1416-1423. Abstract Article

Tax, F.E., and Thomas, J.H. (1994). Cell-cell interactions. Receiving signals in the nematode embryo. Curr. Biol. 4, 914-916. Abstract Article

Tax, F.E., Thomas, J.H., Ferguson, E.L., and Horvitz, H.R. (1997). Identification and characterization of genes that interact with lin-12 in Caenorhabditis elegans. Genetics 147, 1675-1695. Abstract

Thinakaran, G., Borchelt, D.R., Lee, M.K., Slunt, H.H., Spitzer, L., Kim, G., Ratovitsky, T., Davenport, F., Nordstedt, C., Seeger, M., et al. (1996). Endoproteolysis of presenilin 1 and accumulation of processed derivatives in vivo. Neuron 17, 181-190. Abstract Article

Urano, F., Calfon, M., Yoneda, T., Yun, C., Kiraly, M., Clark, S.G., and Ron, D. (2002). A survival pathway for Caenorhabditis elegans with a blocked unfolded protein response. J. Cell Biol. 158, 639-646. Abstract Article

Wen, C., and Greenwald, I. (1999). p24 proteins and quality control of LIN-12 and GLP-1 trafficking in Caenorhabditis elegans. J. Cell Biol. 145, 1165-1175. Article

Wen, C., Levitan, D., Li, X., and Greenwald, I. (2000). spr-2, a suppressor of the egg-laying defect caused by loss of sel-12 presenilin in Caenorhabditis elegans, is a member of the SET protein subfamily. Proc. Natl. Acad. Sci. USA 97, 14524-14529. Abstract Article

Wen, C., Metzstein, M.M., and Greenwald, I. (1997). SUP-17, a Caenorhabditis elegans ADAM protein related to Drosophila KUZBANIAN, and its role in LIN-12/NOTCH signalling. Development 124, 4759-4767. Abstract

Westlund, B., Parry, D., Clover, R., Basson, M., and Johnson, C.D. (1999). Reverse genetic analysis of Caenorhabditis elegans presenilins reveals redundant but unequal roles for sel-12 and hop-1 in Notch-pathway signaling. Proc. Natl. Acad. Sci. USA 96, 2497-2502. Abstract Article

Wharton, K.A., Johansen, K.M., Xu, T., and Artavanis-Tsakonas, S. (1985). Nucleotide sequence from the neurogenic locus notch implies a gene product that shares homology with proteins containing EGF-like repeats. Cell 43, 567-581. Abstract Article

Wilkinson, H.A., Fitzgerald, K., and Greenwald, I. (1994). Reciprocal changes in expression of the receptor lin-12 and its ligand lag-2 prior to commitment in a C. elegans cell fate decision. Cell 79, 1187-1198. Article

Wu, G., Hubbard, E.J., Kitajewski, J.K., and Greenwald, I. (1998). Evidence for functional and physical association between Caenorhabditis elegans SEL-10, a Cdc4p-related protein, and SEL-12 presenilin. Proc. Natl. Acad. Sci. USA 95, 15787-15791. Article 
Wu, G., Lyapina, S., Das, I., Li, J., Gurney, M., Pauley, A., Chui, I., Deshaies, R.J., and Kitajewski, J. (2001). SEL-10 is an inhibitor of notch signaling that targets notch for ubiquitin-mediated protein degradation. Mol. Cell Biol. 21, 7403-7415. Abstract Article

Wu, L., Aster, J.C., Blacklow, S.C., Lake, R., Artavanis-Tsakonas, S., and Griffin, J.D. (2000). MAML1, a human homologue of Drosophila mastermind, is a transcriptional co-activator for NOTCH receptors. Nat. Genet. 26, 484-489. Abstract Article

Yochem, J., and Greenwald, I. (1989). glp-1 and lin-12, genes implicated in distinct cell-cell interactions in $C$. elegans, encode similar transmembrane proteins. Cell 58, 553-563. Abstract Article

Yochem, J., Weston, K., and Greenwald, I. (1988). The Caenorhabditis elegans lin-12 gene encodes a transmembrane protein with overall similarity to Drosophila Notch. Nature 335, 547-550. Abstract Article

Yoo, A.S., Bais, C., and Greenwald, I. (2004). Crosstalk between the EGFR and LIN-12/Notch pathways in $C$. elegans vulval development. Science 303, 663-666. Abstract Article

Yu, G., Nishimura, M., Arawaka, S., Levitan, D., Zhang, L., Tandon, A., Song, Y.Q., Rogaeva, E., Chen, F., Kawarai, T., et al. (2000). Nicastrin modulates presenilin-mediated notch/glp-l signal transduction and BAPP processing. Nature 407, 48-54. Abstract Article

All WormBook content, except where otherwise noted, is licensed under a Creative Commons Attribution License. 